\title{
Comparative Effectiveness of High-Dose Versus Standard-Dose Influenza Vaccine Among Patients Receiving Maintenance Hemodialysis
}

\author{
Anne M. Butler, J. Bradley Layton, Vikas R. Dharnidharka, John M. Sahrmann, Marissa J. Seamans, \\ David J. Weber, and Leah J. McGrath
}

\begin{abstract}
Rationale \& Objective: Studies of patients on maintenance dialysis therapy suggest that standard-dose influenza vaccine (SDV) may not prevent influenza-related outcomes. Little is known about the comparative effectiveness of SDV versus high-dose influenza vaccine (HDV) in this population.
\end{abstract}

Study Design: Cohort study using data from the US Renal Data System.

Setting \& Participants: 507,552 adults undergoing in-center maintenance hemodialysis between the 2010 to 2011 and 2014 to 2015 influenza seasons.

Exposures: SDV and HDV.

Outcomes: All-cause mortality, hospitalization due to influenza or pneumonia, and influenza-like illness during the influenza season.

Analytic Approach: Patients were eligible for inclusion in multiple yearly cohorts; thus, our unit of analysis was the influenza patient-season. To examine the relationship between vaccine dose and effectiveness outcomes, we estimated risk differences and risk ratios using propensity score weighting of Kaplan-Meier functions, accounting for a wide range of patient- and facility-level characteristics. For nonmortality outcomes, we used competing-risk methods to account for the high mortality rate in the dialysis population.

Results: Within 225,215 influenza patientseasons among adults 65 years and older, 97.4\% received SDV and $2.6 \%$ received HDV. We observed similar risk estimates for HDV and SDV recipients for mortality (risk difference, $-0.08 \% ; 95 \% \mathrm{Cl},-0.85 \%$ to $0.80 \%$ ), hospitalization due to influenza or pneumonia (risk difference, 0.15\%; 95\% $\mathrm{Cl},-0.69 \%$ to $0.93 \%$ ), and influenza-like illness (risk difference, $0.00 \% ; 95 \% \mathrm{Cl},-1.50 \%$ to $1.08 \%$ ). Our findings were similar among adults younger than 65 years, as well as within other subgroups defined by influenza season, age group, dialysis vintage, month of influenza vaccination, and vaccine valence.

Limitations: Residual confounding and outcome misclassification.

Conclusions: The HDV does not appear to provide additional protection beyond the SDV against all-cause mortality or influenza-related outcomes for adults undergoing hemodialysis. The additional cost and side effects associated with HDV should be considered when offering this vaccine. Future studies of HDV and other influenza vaccine strategies are warranted.
Complete author and article information provided before references.

Correspondence to

A.M.Butler (anne.butler@ wustl.edu)

Am J Kidney Dis. 75(1):

72-83. Published online

August 1, 2019.

doi: $10.1053 /$

j.ajkd.2019.05.018
Influenza causes substantial morbidity and mortality in patients with end-stage renal disease (ESRD). Patients with ESRD have an impaired innate and adaptive immune system, including defects in complement activation and Band T-cell function, ${ }^{1-3}$ which contribute to increased risks for severe complications from influenza or influenzarelated hospitalizations, mortality, and health care costs compared to the general population. ${ }^{4-7}$ The Centers for Disease Control and Prevention (CDC) has long recommended that patients with ESRD receive annual influenza vaccinations. ${ }^{8,9}$

Currently, several types of influenza vaccines are available in the United States, including the standard-dose (SDV) and high-dose influenza vaccines (HDV). The trivalent - and more recently quadrivalent - inactivated seasonal SDVs have been administered as standard practice in dialysis clinics. In 2009, the US Food and Drug Administration licensed a trivalent HDV for use among adults 65 years and older to induce higher antibody responses and provide better protection from influenza than the SDV. ${ }^{10}$ The HDV contains the same 3 strains as SDV but more antigen (60 vs $15 \mu \mathrm{g}$ per strain) than standard vaccines. Use of the HDV among patients on maintenance hemodialysis has increased slowly over time. ${ }^{11}$

An accumulating body of literature demonstrates that the HDV is more effective than SDVs in preventing influenzarelated medical encounters, hospitalizations, and mortality in the general population of adults 65 years and older. ${ }^{12-18}$ However, some studies of healthy older adults have not demonstrated a benefit of HDV. ${ }^{19,20}$ In the dialysis population, a recent observational study by Miskulin et $\mathrm{al}^{21}$ reported that HDV was more effective than SDV in preventing all-cause hospitalization during the 2016 to 2017 influenza season, but not the 2015 to 2016 season. However, this study was limited by small sample size, adjustment for a small set of potential confounders, and the absence of influenza-related outcomes, warranting additional examination of the effectiveness of the HDV in the dialysis population. 
We sought to compare the clinical effectiveness of HDV versus SDV in preventing all-cause mortality, hospitalization due to influenza or pneumonia, and influenza-like illness (ILI) among patients with chronic kidney failure undergoing maintenance hemodialysis. Because the healthy-user effect and frailty are well-documented challenges of observational studies of influenza vaccine effectiveness in populations with poor health, we used robust methods to account for possible confounding. We also used competing-risk methods to account for the high mortality rate in the dialysis population.

\section{Methods}

\section{Data Source}

We used the US Renal Data System (USRDS) to identify patients with chronic kidney disease receiving in-center maintenance hemodialysis during 2009 to 2015. USRDS is a national registry of patients with ESRD who are eligible for Medicare coverage from the Centers for Medicare \& Medicaid Services (CMS), and it contains routinely reported clinical information from dialysis centers, as well as Medicare claims for inpatient, outpatient, and physician diagnoses and procedures. ${ }^{22}$

\section{Study Design and Patients}

We constructed yearly cohorts for 5 individual influenza seasons: 2010 to 2011, 2011 to 2012, 2012 to 2013, 2013 to 2014, and 2014 to 2015 . The index dates were anchored on the date of influenza vaccination (Fig 1), which was required to be administered before the start date of the influenza season. Eligible patients included adults 18 years or older with ESRD and Medicare as a primary insurance payer who had started hemodialysis at least 9 months before the index date, to allow for a 3month Medicare enrollment period before the 6-month baseline period. We further required continuous hemodialysis receipt for the 3 months immediately before vaccination. Baseline covariates were ascertained during the 6-month baseline period before the index date. The follow-up period began on the start date of the influenza season. The start and end dates of each influenza season were defined using national influenza surveillance data from the CDC. ${ }^{23-27}$ Specifically, the start and end of each season were defined as the midpoint of the first week during which $>10 \%$ and $<10 \%$, respectively, of national culture isolates were positive for influenza (Table S1). The primary analysis was conducted in adults 65 years and older; a secondary analysis was conducted in adults younger than 65 years. Patients could be eligible for inclusion in multiple yearly cohorts.

\section{Exposure Assessment}

Influenza vaccines were classified as HDV, SDV, or unknown (Table S2), based on outpatient, physician, and inpatient procedure coding in billing claims for influenza vaccine or influenza vaccine administration. We searched for Current Procedural Terminology, Healthcare Common Procedure Coding System (HCPCS), and International Classification of Diseases, Ninth Revision, Clinical Modification (ICD-9-CM) codes assigned between August 1 and the end of each influenza season. We assumed that multiple codes within a 7-day period were duplicate codes for the same vaccination and therefore 2 codes for the same vaccine dose were collapsed, whereas 2 codes for a known and unknown dose were categorized as the known dose. Codes within 7 days for different known doses were considered separate doses. Codes spaced by greater than 7 days were also considered separate doses.

\section{Outcome Assessment}

We examined 3 primary clinical outcomes: all-cause mortality, the first occurrence of hospitalization for influenza or pneumonia, and the first occurrence of ILI. All-cause mortality was assessed using the CMS ESRD Death Notification Form. Because laboratory-confirmed influenza status is not available in the claims data, we used 2 levels of claims-based influenza definitions from diagnosis coding: (1) hospitalization for influenza or pneumonia and (2) inpatient or outpatient ILI (see Table S3 for code lists). We performed sensitivity analysis of ILI with a narrower definition, as done previously. ${ }^{28,29}$

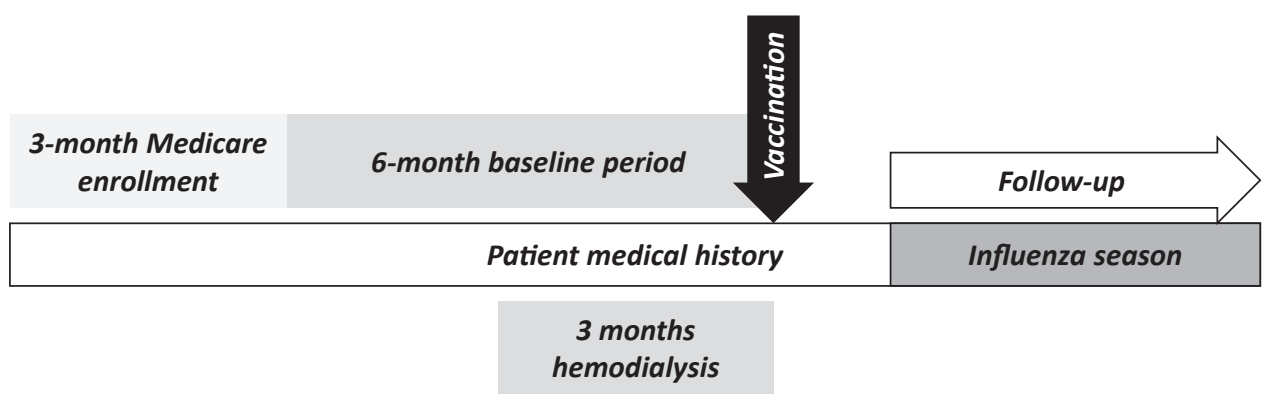

Figure 1. Study design schematic of inclusion criteria and follow-up time for each yearly cohort of adult patients undergoing maintenance hemodialysis. The index date was anchored on the date of influenza vaccination, which was required to be administered before the start date of the influenza season. Follow-up began on the start date of the influenza season. 
Table 1. Characteristics of the 225,215 Unique Influenza PatientSeasons Among US Adults 65 Years and Older Undergoing Maintenance Hemodialysis, by Influenza Vaccine Dose

\begin{tabular}{|c|c|c|}
\hline Characteristic & $\begin{array}{l}\text { HDV } \\
(n=5,776 ; 2.6 \%)\end{array}$ & $\begin{array}{l}\text { SDV } \\
(n=219,439 ; 97.4 \%)\end{array}$ \\
\hline Mean age, y & $75.8 \pm 6.9$ & $74.6 \pm 7.0$ \\
\hline Male sex & $3,064(53.0 \%)$ & $111,827(51.0 \%)$ \\
\hline \multicolumn{3}{|l|}{ Race } \\
\hline White & $4,378(75.8 \%)$ & $134,917(61.5 \%)$ \\
\hline Black & $1,125(19.5 \%)$ & $71,408(32.5 \%)$ \\
\hline Other & $273(4.7 \%)$ & $13,114(6.0 \%)$ \\
\hline \multicolumn{3}{|l|}{ Cause of ESRD } \\
\hline Diabetes & $2,524(43.7 \%)$ & $103,702(47.3 \%)$ \\
\hline Hypertension & $1,898(32.9 \%)$ & 71,973 (32.8\%) \\
\hline GN & $443(7.7 \%)$ & $14,708(6.7 \%)$ \\
\hline Other & $911(15.8 \%)$ & $29,056(13.2 \%)$ \\
\hline \multicolumn{3}{|l|}{ Region } \\
\hline Northeast & $928(16.1 \%)$ & $37,869(17.3 \%)$ \\
\hline South & 1,959 (33.9\%) & $97,158(44.3 \%)$ \\
\hline West & $801(13.9 \%)$ & $39,280(17.9 \%)$ \\
\hline Midwest & $2,088(36.1 \%)$ & $45,132(20.6 \%)$ \\
\hline $\begin{array}{l}\text { Dual-eligible for } \\
\text { Medicaid }\end{array}$ & $1,338(23.2 \%)$ & 76,492 (34.9\%) \\
\hline \multicolumn{3}{|l|}{ Dialysis vintage } \\
\hline$<1 \mathrm{y}$ & $420(7.3 \%)$ & $11,394(5.2 \%)$ \\
\hline $1-2 y$ & $2,512(43.5 \%)$ & $76,237(34.7 \%)$ \\
\hline $3-4 y$ & $1,348(23.3 \%)$ & $54,441(24.8 \%)$ \\
\hline $5-9 y$ & $1,172(20.3 \%)$ & $58,560(26.7 \%)$ \\
\hline$\geq 10 y$ & $324(5.6 \%)$ & $18,807(8.6 \%)$ \\
\hline
\end{tabular}

No. of days

hospitalized in

last month

\begin{tabular}{lll}
\hline 0 & $5,391(93.3 \%)$ & $205,549(93.7 \%)$ \\
\hline $1-6$ & $257(4.4 \%)$ & $9,358(4.3 \%)$ \\
\hline$\geq 7$ & $128(2.2 \%)$ & $4,532(2.1 \%)$ \\
\hline Skilled nursing facility & $133(2.3 \%)$ & $5,911(2.7 \%)$
\end{tabular}

in last mo

Comorbid conditions

\begin{tabular}{lll}
\hline Cancer & $895(15.5 \%)$ & $25,393(11.6 \%)$ \\
\hline COPD & $898(15.5 \%)$ & $29,177(13.3 \%)$ \\
\hline Diabetes & $3,666(63.5 \%)$ & $141,026(64.3 \%)$ \\
\hline Hypertension & $3,896(67.5 \%)$ & $139,110(63.4 \%)$ \\
\hline $\begin{array}{l}\text { Ischemic heart } \\
\text { disease }\end{array}$ & $3,613(62.6 \%)$ & $122,555(55.8 \%)$ \\
\hline $\begin{array}{l}\text { Liver disease } \\
\text { Frailty indicators }\end{array}$ & $441(7.6 \%)$ & $10,511(4.8 \%)$ \\
\hline $\begin{array}{l}\text { Ambulance/life } \\
\text { support }\end{array}$ & $1,328(23.0 \%)$ & $55,171(25.1 \%)$ \\
\hline $\begin{array}{l}\text { Difficulty walking } \\
\text { Mobility aids }\end{array}$ & $714(12.4 \%)$ & $28,605(13.0 \%)$ \\
\hline $\begin{array}{l}\text { Skin ulcer } \\
\text { (decubitus) }\end{array}$ & $526(9.1 \%)$ & $22,011(10.0 \%)$ \\
\hline $\begin{array}{l}\text { Use of oxygen } \\
\text { Weakness }\end{array}$ & $675(10.0 \%)$ & $19,792(9.0 \%)$ \\
\hline
\end{tabular}

(Continued)

\section{Covariates}

Our analyses included a wide range of covariates identified during the 6-month baseline period (Fig 1; Table S4). Covariates included demographic characteristics (eg, age,
Table 1 (Cont'd). Characteristics of the 225,215 Unique Influenza Patient-Seasons Among US Adults 65 Years and Older Undergoing Maintenance Hemodialysis, by Influenza Vaccine Dose

\begin{tabular}{lll}
\hline $\begin{array}{l}\text { Characteristic } \\
\text { Screening tests/ }\end{array}$ & $\begin{array}{l}\text { HDV } \\
\text { (n= 5,776; 2.6\%) }\end{array}$ & $\begin{array}{l}\text { SDV } \\
\text { (n= 219,439; 97.4\%) }\end{array}$ \\
\begin{tabular}{l} 
prevention \\
\hline $\mathrm{HbA}_{1 \mathrm{c}}$ test
\end{tabular} & $3,155(54.6 \%)$ & $129,109(58.8 \%)$ \\
\hline $\begin{array}{l}\text { Cancer screening } \\
\text { Diabetic eye }\end{array}$ & $241(14.6 \%)$ & $30,543(13.9 \%)$ \\
$\begin{array}{l}\text { examination } \\
\begin{array}{l}\text { Hepatitis B vaccine/ } \\
\text { titer }\end{array}\end{array}$ & $1,288(22.3 \%)$ & $42,217(19.2 \%)$ \\
$\begin{array}{l}\text { Lipid test } \\
\begin{array}{l}\text { Pneumococcal } \\
\text { vaccine }\end{array}\end{array}$ & $1,991(34.5 \%)$ & $66,996(30.5 \%)$ \\
\hline
\end{tabular}

Abbreviations: COPD, chronic obstructive pulmonary disease; ESRD, end-stage renal disease; GN, glomerulonephritis; $\mathrm{HbA}_{1 \mathrm{c}}$, hemoglobin $\mathrm{A}_{1 \mathrm{c}} ; \mathrm{HDV}$, high-dose vaccine; SDV, standard-dose vaccine.

${ }^{2}$ Defined as use of walker, wheelchair, or modified bathroom equipment.

sex, race, dual-eligible for Medicaid, region, and year), clinical characteristics (eg, cause of ESRD and years on dialysis), dialysis facility characteristics (eg, affiliation, type, profit status, and size), and several comorbid conditions and procedures captured using ICD-9-CM and HCPCS codes. To account for potential confounding by frailty $^{30,31}$ and healthy-user bias, ${ }^{32,33}$ we also included covariates on preventive health services (eg, other vaccinations and health screenings), health care utilization (eg, skilled nursing days and hospital days), and frailty indicators (eg, oxygen use and mobility aids). We categorized the timing of vaccine administration as vaccination in August or September versus October through the start of influenza season.

\section{Statistical Analysis}

Patients could be eligible for inclusion in multiple yearly cohorts; thus, our unit of analysis was the influenza patient-season. To examine the relationship between vaccine dose and effectiveness outcomes, we estimated crude and weighted cumulative risk functions using KaplanMeier methods, accounting for the competing risk for death (for the nonmortality outcomes of ILI and hospitalization due to influenza or pneumonia). ${ }^{34-36} \mathrm{We}$ addressed potential confounding due to differences in observed covariates between HDV and SDV recipients by using propensity score weighting methods. Specifically, we applied standardized mortality ratio weights to the Kaplan-Meier estimator. We estimated the predicted probability of receiving HDV, or the propensity score (ps), using logistic regression with a priori selected potential confounders as predictors (Table S4). Age was modeled using restricted quadratic splines with 4 knots. Standardized mortality ratio weights for SDV recipients were calculated as ps/(1-ps); HDV recipients remained unweighted. ${ }^{37}$ We plotted the standardized mean differences 
of baseline covariates in the unweighted and standardized mortality ratio-weighted populations to determine whether weighting the population reduced imbalances of observed covariates and made the treatment groups more exchangeable. ${ }^{38}$ We stratified models by influenza season, valence, age, dialysis vintage (in years), and timing of influenza vaccination; weights were created separately for each subgroup analysis. Covariate balance was assessed separately for each subgroup analysis. Patients receiving influenza vaccine before the beginning of the influenza season were followed up from the first day of the influenza season until the first occurrence of the outcome, a competing-risk event (ie, death for nonmortality outcomes), or a censoring event (ie, disenrollment from Medicare Parts A and B, kidney transplantation, switch to peritoneal dialysis, loss to follow-up, receipt of a subsequent influenza vaccine, or end of influenza season). We calculated risk differences and risk ratios using the cumulative risk function estimates at the end of each influenza season. A nonparametric-based bootstrap was used to estimate 95\% confidence intervals (CIs) for daily risk differences and risk ratios between groups. Additionally, we conducted bias analyses to estimate the strength of unmeasured confounders that would be required to mask associations between vaccine type and each primary outcome after adjusting for measured covariates.

This study using deidentified data was considered exempt from human subject review by the Institutional Review Board at Washington University. Analyses were performed using SAS, version 9.4 (SAS Institute), and R Statistical Software, version 3.3. ${ }^{40}$

\section{Results}

After applying inclusion and exclusion criteria, we identified 255,281 eligible adult patients who contributed 507,552 unique influenza patient-seasons (Fig S1). In the primary analysis of adults 65 years and older $(n=225,215$ influenza patient-seasons), SDV made up $97.4 \%$ $(n=219,439)$ of the eligible vaccinations, and HDV was administered in the remaining 2.6\% $(\mathrm{n}=5,776)$. All HDV were trivalent, whereas $76.7 \%$ of SDV were trivalent and $23.3 \%$ were quadrivalent.

Tables 1 and S5 present characteristics of the study population stratified by vaccine dose. Mean age was slightly older among HDV recipients than SDV recipients (75.8 vs 74.6 years). HDV administration was less common among patients who were black or other race, dualeligible for Medicaid, on dialysis for 3 or more years, or residing outside of the Midwest. HDV recipients had a higher prevalence of several comorbid conditions, including ischemic heart disease, chronic obstructive pulmonary disease, cancer, and liver disease. HDV and SDV recipients had a similar prevalence of frailty indicators. HDV recipients were more likely to receive preventive health care such as diabetic eye examinations, lipid testing, and cancer screenings. Some characteristics of the HDV 
recipients changed over time (eg, dialysis vintage; Table S6). The distribution of observed covariates was well balanced after propensity score weighting, as indicated by standardized mean differences $<10 \%$ in the weighted population. ${ }^{41}$ Figure S2 presents these results for the primary analysis.

The counts of outcome events, censoring events, competing-risk events, and person-time at risk are presented for each outcome in Table 2. Among SDV recipients, crude risks for death and hospitalization for pneumonia or influenza were similar $(8.7 \%$ and $7.6 \%$, respectively); in contrast, the risk for ILI was more than 3 times higher (28.1\%).

For each outcome, we observed similar weighted risks for HDV and SDV recipients throughout the influenza season, accounting for the competing risk for death for nonmortality outcomes (Fig 2). In the weighted analyses, we observed similar associations between vaccine dose and risk for mortality (risk difference, $-0.08 \%$; 95\% CI, $-0.85 \%$ to $0.80 \%$ ), hospitalization due to influenza or pneumonia (risk difference, $0.15 \%$; $95 \% \mathrm{CI},-0.69 \%$ to $0.93 \%$ ), and ILI (risk difference, $0.00 \%$; $95 \% \mathrm{CI},-1.50 \%$ to $1.08 \%$ ).

Results did not change appreciably in the analysis with the restricted ILI definition (Table S7). Estimates on the relative scale followed similar patterns (Table 2).

Within subgroups, risks for mortality, hospitalization for pneumonia or influenza, and ILI were generally similar between HDV and SDV recipients throughout the influenza season, apart from a few notable exceptions (Figs 3-5;
Tables S8-S10). First, we observed higher risk for hospitalization among HDV versus SDV recipients in the 2010 to 1011 season (risk difference, 2.85\%; 95\% CI, 0.59\%$5.86 \%)$, but not for any other season. Also, for both the hospitalization and ILI outcomes, we observed higher risk among HDV versus SDV recipients who were aged 65 to 74 years, lower risk among those aged 75 to 84 years, and no difference in risk between those younger than 65 or 85 years and older.

In the bias analyses, we quantified the possibility that our observed null associations may be explained by an unmeasured confounding variable. We observed that the exposure-confounder relative risk and the confounderoutcome relative risk must have been at least as large as $1.4,1.5$, and 1.3 for mortality, hospitalization due to influenza or pneumonia, and ILI, respectively, to have shifted the corresponding weighted upper confidence limit across the null (Tables S11-S13).

\section{Discussion}

We conducted a large comparative study of the effectiveness of the HDV versus SDV among adults with chronic kidney failure undergoing maintenance hemodialysis in the United States. In the overall population, we observed similar risks for all-cause mortality, hospitalization due to influenza or pneumonia, and ILI among patients who received HDV compared with those who received SDV. Our findings were generally consistent across subgroups of
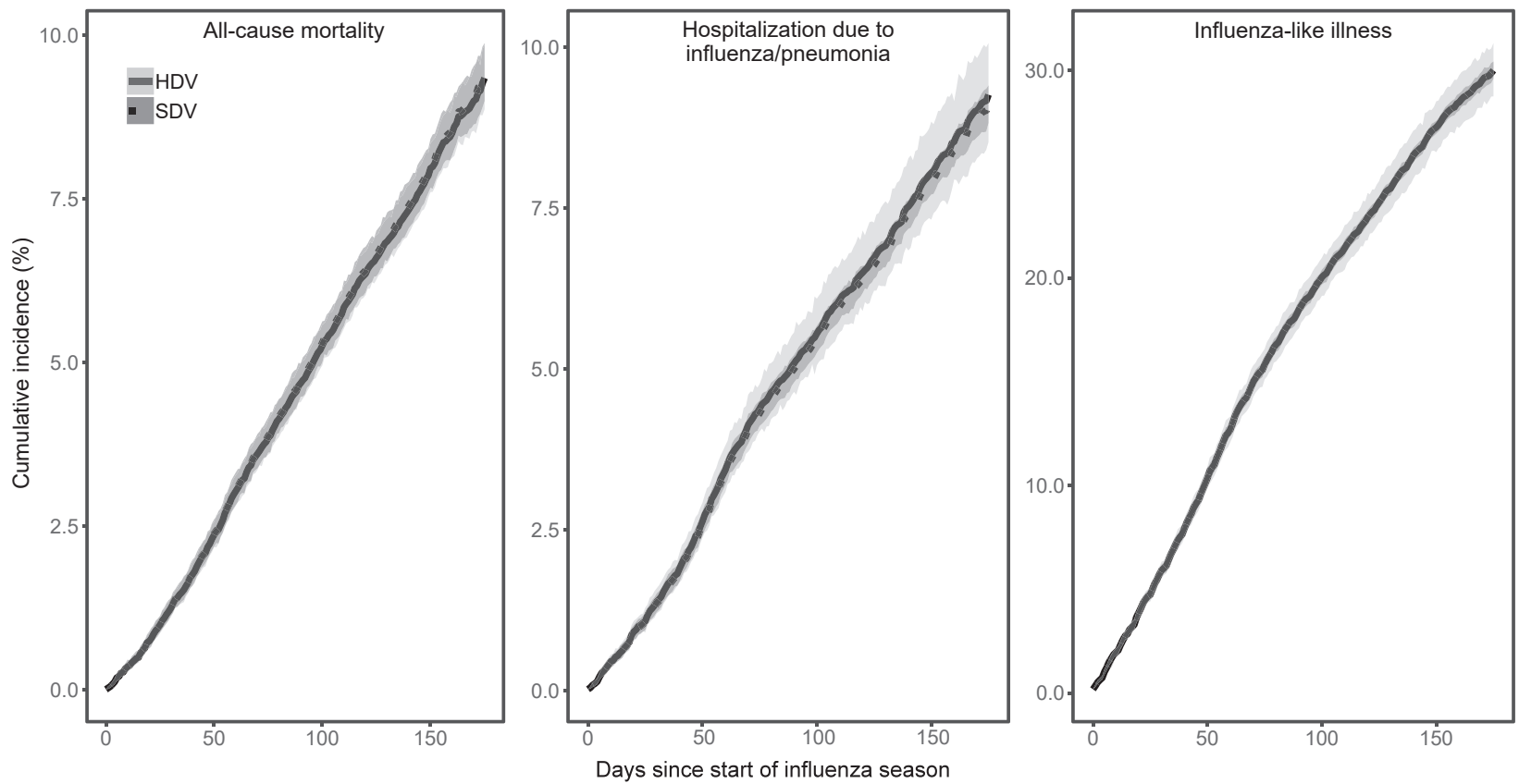

Figure 2. Cumulative risk and $95 \%$ confidence interval estimates of mortality, hospitalization for pneumonia or influenza, and influenza-like illness, accounting for the competing risk for death (for nonmortality outcomes), pooled across 5 influenza seasons (2010/2011, 2011/2012, 2012/2013, 2013/2014, 2014/2015). Cumulative risk was estimated using standardized mortality ratio-weighted Kaplan-Meier functions. The scales of the $y$-axes differ by outcome. Abbreviations: HDV, high-dose influenza vaccine; SDV, standard-dose influenza vaccine. 


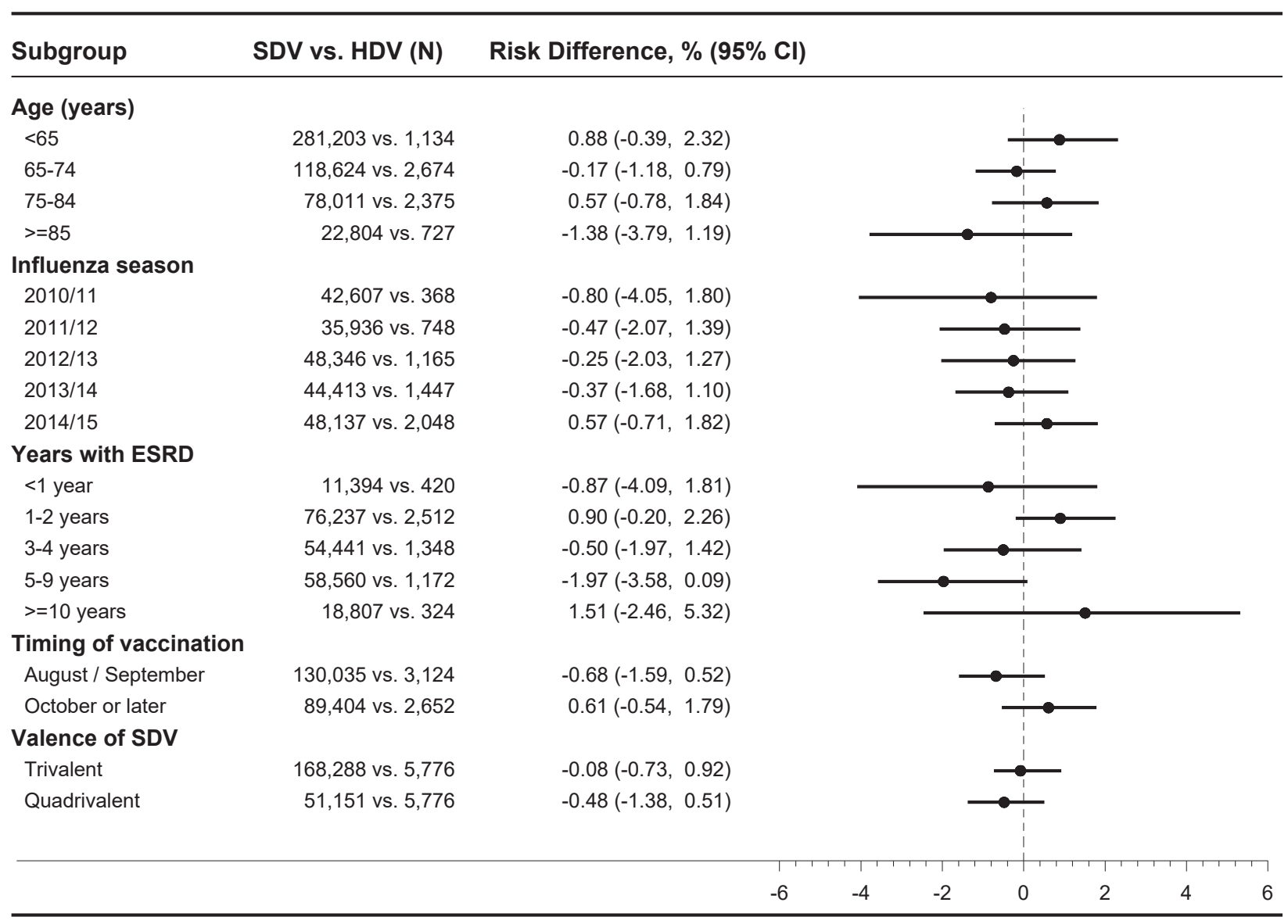

Figure 3. The risk for mortality among patients who received the high-dose (HDV) versus standard-dose (SDV) influenza vaccine, by subgroup. Risk differences were calculated as the difference between weighted cumulative risk functions, with bootstrap confidence intervals (Cls). Analyses were performed in adults 65 years and older, with the exception of the age-stratified analyses. Abbreviation: ESRD, end-stage renal disease.

influenza season, age group, years on dialysis, timing of influenza vaccination, and valence. We observed differences between HDV and SDV in a few subgroups, but the patterns were not consistent across influenza seasons or age groups. Associations between a potential unmeasured confounder and our 3 outcomes would have required associations that are stronger than our measured confounder-outcome associations to have masked preventive effects.

Consideration of the results of comparative effectiveness studies of preventive therapies requires careful thought about the tradeoffs of benefits versus costs. Due to the substantial influenza burden in the dialysis population, even small improvements in vaccine effectiveness could be sufficient for recommending a specific type of annual influenza vaccination in this population. However, increased risks for side effects and increased costs must also be considered when weighing the costbenefit of implementing a new vaccination strategy. Our results suggest that the HDV does not provide additional benefit beyond the SDV on a population level. Additionally, the excess cost of the HDV is about $\$ 33$ per dose, $^{42}$ incurring an additional cost burden of $\$ 16.9$ million under the assumption that all 511,000 patients on dialysis would be vaccinated with $\mathrm{HDV} .{ }^{43} \mathrm{HDV}$ is also more reactogenic, causing more frequent side effects, although most of these adverse events are minor (eg, injection-site reactions, systemic adverse events, and gastrointestinal events). ${ }^{12,19,44-48}$ Safety data for the HDV are limited to the healthy adult population; a comparison of the safety of HDV versus SDV in patents receiving dialysis is needed.

Previous observational studies have demonstrated that SDVs are ineffective or minimally effective at reducing allcause mortality or influenza-related hospitalizations in patients receiving dialysis ${ }^{29,49}$ despite reasonably robust antibody responses regarded as protective in 30\% to $90 \%$ of vaccine recipients. ${ }^{50-55}$ Our work extends these findings by clarifying that the HDV does not provide additional protection beyond the SDV against all-cause mortality or influenza-related outcomes, although the HDV elicits greater antibody responses than SDV in patients receiving dialysis. ${ }^{56}$ These observations highlight the importance of postlicensure clinical studies that extend beyond 


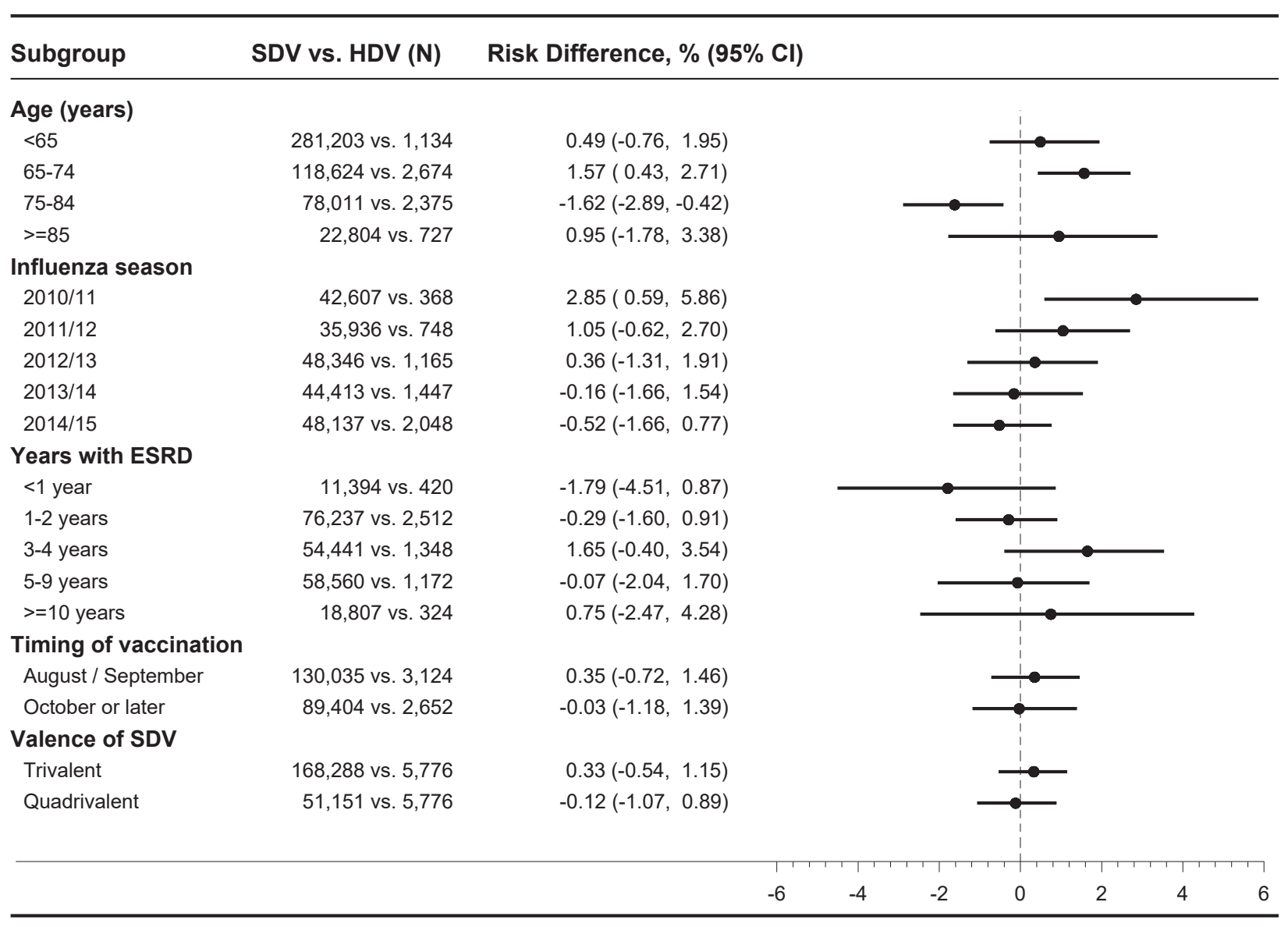

Figure 4. The risk for hospitalization due to influenza/pneumonia among patients who received the high-dose (HDV) versus standard-dose (SDV) influenza vaccine, by subgroup. Risk differences were calculated as the difference between weighted cumulative risk functions, accounting for the competing risk for death, with bootstrap confidence intervals (Cls). Analyses were performed in adults 65 years and older, with the exception of the age-stratified analyses. Abbreviation: ESRD, end-stage renal disease.

immunogenicity-related outcomes to incorporate clinical outcomes.

Our conclusions differ somewhat from an observational study using data from a not-for-profit dialysis provider in the United States. ${ }^{21}$ Similar to our study, Miskulin et $\mathrm{al}^{21}$ reported similar rates of all-cause mortality among HDV versus SDV recipients. In contrast to our study, Miskulin et al reported lower rates of all-cause hospitalization among HDV versus SDV recipients, though their finding was restricted to the 2016 to 2017 influenza season and not the 2015 to 2016 season. The $95 \%$ CIs for the preventive effect of HDV (hazard ratio, 0.93; 95\% CI, 0.86$1.00)$ included the null value, whereas the effect estimate moved down and away from the null in the subgroup analysis restricted to patients 65 years and older (hazard ratio, $0.88 ; 95 \%$ CI, 0.79-0.97). Other stratified analyses suggested similar protection among HDV and SDV recipients.

Our results are not directly comparable with the previous study given several differences. Specifically, Miskulin et al included later seasons (2015/2016 and 2016/2017), patients undergoing peritoneal dialysis, and a smaller sample size $(\mathrm{N}=19,157)$. Furthermore, the previous study did not incorporate influenzarelated outcomes or use competing-risks methodology to account for the high mortality in the dialysis population.

Perhaps most importantly, the analysis by Miskulin et al accounted for a limited set of potential confounders and therefore their effect estimates may be subject to residual confounding bias. In contrast, our study accounted for potential confounding by frailty ${ }^{30,31}$ and the healthy-user bias $^{32,33}$ through adjustment for a comprehensive set of individual- and facility-level potential confounders. It is well established that observational studies of the effectiveness of preventive interventions such as influenza vaccination in elderly or sick populations can be highly subject to confounding by frailty and health care-seeking behavior because those who receive the treatment generally have higher anticipation of benefit by providers (eg, longer predicted life expectancies) or stronger personal motivation to receive care (eg, better access to health care, higher socioeconomic status, and less frailty). ${ }^{49,57-60}$ Often, this confounding is difficult to control and results in artificially protective adjusted effect estimates due to residual confounding. 


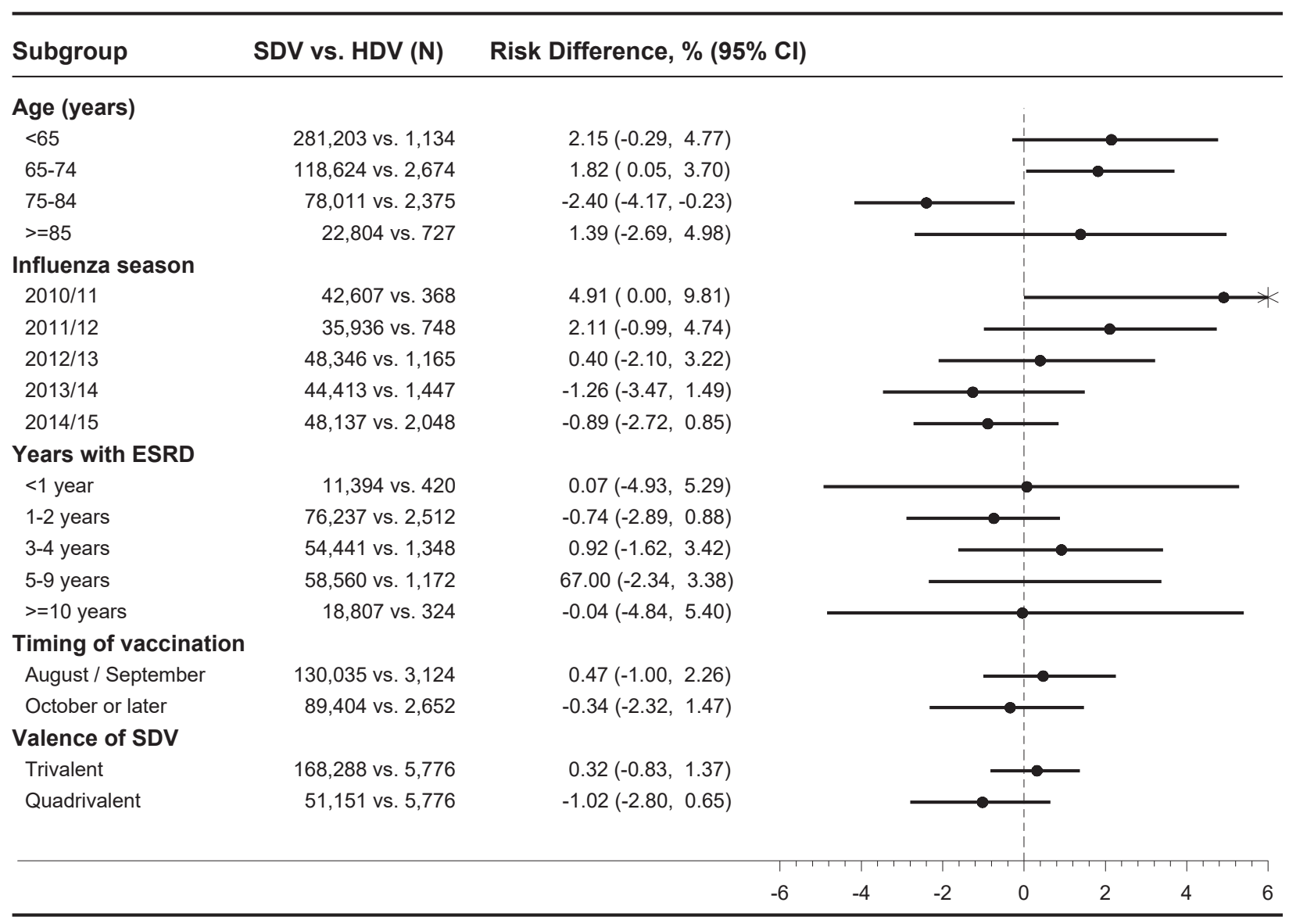

Figure 5. The risk for influenza-like illness among patients who received the high-dose (HDV) versus standard-dose (SDV) influenza vaccine, by subgroup. Risk differences were calculated as the difference between weighted cumulative risk functions, accounting for the competing risk for death, with bootstrap confidence intervals (Cls). Analyses were performed in adults 65 years and older, with the exception of the age-stratified analyses. Abbreviation: ESRD, end-stage renal disease.

We were concerned that this phenomenon might also be present when comparing HDV, a newer, more expensive, specialized vaccine, to the SDV. However, that is not what we observed. Instead, older individuals receiving HDV tended to be sicker and the crude effect estimate was above the null, suggesting that the confounding was not due to the healthy-user phenomenon. This is supported by our previous work, in which we demonstrated that comparisons of HDV to SDV among adult patients receiving dialysis are less subject to confounding by the healthy-user bias than comparisons of vaccinated versus unvaccinated persons. ${ }^{61}$ However, despite our attempts, the possibility of unmeasured confounding remains.

Our findings should be interpreted within the context of several factors that affect vaccine effectiveness, including influenza virus circulation, serologic vaccine match, and seasonal influenza severity. ${ }^{62}$ Our study period included a range of seasonal influenza severity (1 low severity, 2 moderate, and 2 high seasons) as measured in the overall population of older adults in the United States. ${ }^{63}$ These severity metrics correlated with the observed seasonal risk for clinical outcomes in our analyses. Also, influenza A
(H3N2) viruses, which are associated with lower vaccine effectiveness, were the predominant strains during all influenza seasons in our study, with the exception of 2013 to $2014 .{ }^{23-27}$ Compared to influenza A (H1N1) or influenza B viruses, H3N2 viruses have more frequently undergone antigenic change, resulting in differences between the virus components of the influenza vaccine and circulating influenza viruses. ${ }^{64}$ However, the serologic vaccine match, the proportion of circulating influenza strains that matched the vaccine, was relatively high during 3 of the 4 seasons with H3N2 as the predominant strain (ie, 97\%, $80 \%, 86 \%$, and $40 \%$ for seasons $2010 / 2011,2011 / 2012$, $2012 / 2013$, and $2014 / 2015$, respectively), ${ }^{23-27}$ indicating similarity between the influenza viruses in the vaccine and circulating in the community.

Our results are subject to several limitations. First, our observational study design did not involve randomization of the exposure and therefore our effect estimates are potentially subject to confounding by unobserved differences between exposure groups. However, we attempted to control confounding through restriction of the study population (ie, by vaccination status and age), as well as 
analytic adjustment for a rich set of covariates. It is noteworthy that we did not account for individual dialysis facilities within our regression models; however, our analysis accounted for dialysis center-level characteristics, several of which were strong predictors of HDV receipt. ${ }^{11}$

Second, our analysis only accounted for baseline characteristics measured before vaccination. However, this approach is supported by a previous study of influenza vaccine effectiveness among patients receiving hemodialysis that demonstrated that accounting for time-varying confounders of health status measured from both clinical and claims data did not reduce bias. ${ }^{49}$

Third, our study design required survival until 9 months after dialysis initiation; therefore, our results may not generalize to all incident patients. Fourth, in the absence of laboratory-confirmed influenza outcomes, we used claims-based definitions. Thus, outcome misclassification is a possibility, though we used sensitivity analyses to explore the impact of the definitions on our findings. Fifth, some subgroup analyses may have had limited ability to detect a true difference between HDV and SDV given small numbers of HDV recipients. Sixth, the start date of follow-up was based on national influenza data and therefore ignores geographic variation in the timing of influenza seasons.

Last, our study is primarily based on administrative billing claims data, which are collected for administrative and reimbursement purposes, rather than for clinical research. Thus, we were not able to account for certain important clinical factors unavailable in claims data (eg, body mass index, vascular access, and laboratory measurements) that may result in confounding bias, and some of our measures may be subject to misclassification, such as vaccination ascertainment if patients paid out of pocket. However, we expect this misclassification to be unlikely because patients receiving regular in-center hemodialysis have very frequent health care encounters in dialysis clinics, and influenza vaccination is offered to Medicare beneficiaries without a co-pay. Importantly, our use of these data is also a strength because it captures information on a wide variety of health-related diagnoses, procedures, use of durable medical equipment, frailty markers, hospitalizations, and death.

In summary, our large comparative study failed to demonstrate that HDV has superior effectiveness compared to SDV for preventing all-cause mortality and influenzarelated outcomes among patients receiving maintenance hemodialysis. Given the findings of our population-level study, along with the substantially higher cost and sideeffect profile of HDV compared to SDV, it appears that HDV should not conclusively be considered the standard of care at the present time for influenza immunization of patients treated by maintenance hemodialysis. The findings of our population-level study should not be interpreted to discourage influenza vaccination in the dialysis population. Rather, dialysis patients should continue to receive annual influenza immunization per CDC guidelines. ${ }^{8}$ In addition, future studies of alternative strategies (eg, booster doses) and alternative vaccine production technologies (eg, adjuvanted or cell-based vaccines) are warranted because there remains a need for improved influenza prevention efforts in this population.

\section{Supplementary Material}

\section{Supplementary File (PDF)}

Figure S1: Derivation of cohort in the US Renal Data System, 20092015.

Figure S2: Standardized mean differences of patient- and facilitylevel characteristics, before and after propensity score weighting for the primary analysis.

Table S1: Start and end dates of yearly influenza seasons.

Table S2: Definitions of influenza vaccination.

Table S3: Definitions of influenza outcomes.

Table S4: Definitions of covariates.

Table S5: Additional characteristics of the 225,215 unique influenza patient-seasons among US adults $\geq 65$ years undergoing maintenance HD, by influenza vaccine dose, 2010-2015.

Table S6: Characteristics of the 225,215 unique influenza patientseasons among US adults $\geq 65$ years undergoing maintenance HD, by influenza vaccine dose and influenza season, 2010-2015.

Table S7: Estimates of the preventive effect of HDV vs SDV on the restricted sensitivity analysis definition of $\mathrm{ILI}$, accounting for the competing risk of death, among adults $\geq 65$ years, by influenza season.

Table S8: Stratified estimates of the preventive effect of HDV vs SDV on mortality during the influenza season.

Table S9: Stratified estimates of the preventive effect of HDV vs SDV on hospitalization due to influenza or pneumonia during the influenza season, accounting for the competing risk of death.

Table S10: Stratified estimates of the preventive effect of HDV vs SDV on ILI during the influenza season, accounting for the competing risk of death.

Table S11: Bias-corrected risk ratios of the preventive effect of HDV vs SDV on mortality during the influenza season.

Table S12: Bias-corrected risk ratios of the preventive effect of HDV vs SDV on hospitalization due to influenza or pneumonia during the influenza season.

Table S13: Bias-corrected risk ratios of the preventive effect of HDV vs SDV on ILI during the influenza season.

\section{Article Information}

Authors' Full Names and Academic Degrees: Anne M. Butler, $\mathrm{PhD}$, J. Bradley Layton, PhD, Vikas R. Dharnidharka, MD, MPH, John M. Sahrmann, MA, Marissa J. Seamans, PhD, David J. Weber, MD, MPH, and Leah J. McGrath, PhD.

Authors' Affiliations: Division of Infectious Diseases, Department of Internal Medicine (AMB, JMS), and Division of Public Health Sciences, Department of Surgery (AMB), Washington University School of Medicine, St. Louis, MO; RTI Health Solutions, Research Triangle Park, NC (JBL); Division of Pediatric Nephrology, Hypertension and Pheresis, Department of Pediatrics, Washington University School of Medicine, St. Louis, MO (VRD); Department of Mental Health, The Johns Hopkins Bloomberg School of Public Health, Baltimore, MD (MJS); Division of Infectious Diseases, Department of Medicine, University of North 
Carolina at Chapel Hill, Chapel Hill (DJW); and NoviSci, Durham, NC (LJM).

Address for Correspondence: Anne M. Butler, PhD, Division of Infectious Diseases, John T. Milliken Department of Internal Medicine, Washington University School of Medicine, 4523 Clayton Ave, CB 8051, St. Louis, MO 63110. E-mail: anne. butler@wustl.edu

Authors' Contributions: Research idea and study design: AMB, JBL, LJM; data acquisition: AMB; data analysis/interpretation: AMB, JBL, VRD, JMS, MJS, DJW, LJM; statistical analysis: AMB, JMS, MJS; supervision or mentorship: AMB, JBL, VRD, LJM. Each author contributed important intellectual content during manuscript drafting or revision and accepts accountability for the overall work by ensuring that questions pertaining to the accuracy or integrity of any portion of the work are appropriately investigated and resolved.

Support: This project was supported by the US National Institute of Allergy and Infectious Diseases, National Institutes of Health (NIH), through grant award number 1R21Al138385. Dr Butler is supported by a grant from the National Center for Advancing Translational Sciences (NCATS), NIH, under award number KL2 TR002346. Dr Seamans is supported by a grant from the National Institute on Drug Abuse, NIH, under award number T32DA007292. Data programming for this study was conducted by the Center for Administrative Data Research, which is supported in part by the Washington University Institute of Clinical and Translational Sciences grant UL1 TR002345 from NCATS of the $\mathrm{NIH}$, grant number R24 HS19455 through the Agency for Healthcare Research and Quality. The study sponsors did not have any role in study design; collection, analysis, and interpretation of data; writing the report; or the decision to submit the report for publication.

Financial Disclosure: Dr Butler has received investigator-initiated support from Amgen. $\mathrm{Dr}$ Layton is an employee of RTI International, an independent, nonprofit research organization that performs research on behalf of government and commercial clients, including pharmaceutical companies. Dr McGrath is an employee of NoviSci, a data sciences company, and previously was an employee of RTI Health Solutions. The remaining authors declare that they have no relevant financial interests.

Acknowledgements: The authors acknowledge Amber Salter from Washington University for biostatistical advice and Carrie O'Neil from Washington University for editorial assistance.

Disclaimer: The content is solely the responsibility of the authors and does not necessarily represent the official views of the NIH. The data reported here have been supplied by the USRDS. The interpretation and reporting of these data are the responsibility of the authors and in no way should be seen as an official policy or interpretation of the US government.

Peer Review: Received January 8, 2019. Evaluated by 2 external peer reviewers, with direct editorial input from a Statistics/ Methods Editor, an Associate Editor, and the Editor-in-Chief. Accepted in revised form May 23, 2019.

\section{References}

1. Descamps-Latscha B. The immune system in end-stage renal disease. Curr Opin Nephrol Hypertens. 1993;2(6): 883-891.

2. Hauser $A B$, Stinghen $A E$, Kato $S$, et al. Characteristics and causes of immune dysfunction related to uremia and dialysis. Perit Dial Int. 2008;28(suppl 3):S183-S187.
3. Kato S, Chmielewski M, Honda $\mathrm{H}$, et al. Aspects of immune dysfunction in end-stage renal disease. Clin J Am Soc Nephrol. 2008;3(5):1526-1533.

4. Allon M, Depner TA, Radeva M, et al. Impact of dialysis dose and membrane on infection-related hospitalization and death: results of the HEMO Study. J Am Soc Nephrol. 2003;14(7): 1863-1870.

5. Chavers BM, Solid CA, Gilbertson DT, Collins AJ. Infectionrelated hospitalization rates in pediatric versus adult patients with end-stage renal disease in the United States. J Am Soc Nephrol. 2007;18(3):952-959.

6. Naqvi SB, Collins AJ. Infectious complications in chronic kidney disease. Adv Chronic Kidney Dis. 2006;13(3):199-204.

7. Sarnak MJ, Jaber BL. Mortality caused by sepsis in patients with end-stage renal disease compared with the general population. Kidney Int. 2000;58(4):1758-1764.

8. Grohskopf LA, Sokolow LZ, Broder KR, Walter EB, Fry AM, Jernigan DB. Prevention and control of seasonal influenza with vaccines: recommendations of the Advisory Committee on Immunization Practices-United States, 2018-19 influenza season. MMWR Recomm Rep. 2018;67(3):1-20.

9. Eickhoff TC. Immunization against influenza: rationale and recommendations. J Infect Dis. 1971;123(4):446-454.

10. Centers for Disease Control and Prevention. Licensure of a high-dose inactivated influenza vaccine for persons aged >or=65 years (Fluzone High-Dose) and guidance for use United States, 2010. MMWR Morb Mortal Wkly Rep. 2010;59(16):485-486.

11. McGrath LJ, Layton JB, Krueger WS, Kshirsagar AV, Butler AM. High-dose influenza vaccine use among patients receiving hemodialysis in the United States, 2010-2013. Vaccine. 2018;36(41):6087-6094.

12. Wilkinson K, Wei Y, Szwajcer A, et al. Efficacy and safety of high-dose influenza vaccine in elderly adults: a systematic review and meta-analysis. Vaccine. 2017;35(21):2775-2780.

13. DiazGranados CA, Dunning AJ, Kimmel M, et al. Efficacy of high-dose versus standard-dose influenza vaccine in older adults. N Engl J Med. 2014;371(7):635-645.

14. DiazGranados CA, Dunning AJ, Robertson CA, Talbot HK, Landolfi V, Greenberg DP. Efficacy and immunogenicity of highdose influenza vaccine in older adults by age, comorbidities, and frailty. Vaccine. 2015;33(36):4565-4571.

15. Izurieta HS, Thadani N, Shay DK, et al. Comparative effectiveness of high-dose versus standard-dose influenza vaccines in US residents aged 65 years and older from 2012 to 2013 using Medicare data: a retrospective cohort analysis. Lancet Infect Dis. 2015;15(3):293-300.

16. Shay DK, Chillarige $Y$, Kelman J, et al. Comparative effectiveness of high-dose versus standard-dose influenza vaccines among US Medicare beneficiaries in preventing postinfluenza deaths during 2012-2013 and 2013-2014. J Infect Dis. 2017;215(4):510-517.

17. Richardson DM, Medvedeva EL, Roberts CB, Linkin DR. Comparative effectiveness of high-dose versus standard-dose influenza vaccination in community-dwelling veterans. Clin Infect Dis. 2015;61(2):171-176.

18. Young-Xu Y, Van Aalst R, Mahmud SM, et al. Relative vaccine effectiveness of high-dose versus standard-dose influenza vaccines among Veterans Health Administration patients. J Infect Dis. 2018;217(11):1718-1727.

19. DiazGranados CA, Dunning AJ, Jordanov E, Landolfi V, Denis M, Talbot HK. High-dose trivalent influenza vaccine compared to standard dose vaccine in elderly adults: safety, 
immunogenicity and relative efficacy during the 2009-2010 season. Vaccine. 2013;31(6):861-866.

20. DiazGranados CA, Robertson CA, Talbot HK, Landolfi V, Dunning AJ, Greenberg DP. Prevention of serious events in adults 65 years of age or older: a comparison between highdose and standard-dose inactivated influenza vaccines. Vaccine. 2015;33(38):4988-4993.

21. Miskulin DC, Weiner DE, Tighiouart $H$, et al. High-dose seasonal influenza vaccine in patients undergoing dialysis. Clin J Am Soc Nephrol. 2018;13(11):1703-1711.

22. Saran R, Li Y, Robinson B, et al. US Renal Data System 2015 annual data report: epidemiology of kidney disease in the United States. Am J Kidney Dis. 2016;67(3)(suppl 1):S1S390.

23. Centers for Disease Control and Prevention. Update: influenza activity-United States, 2010-11 season, and composition of the 2011-12 influenza vaccine. MMWR Morb Mortal Wkly Rep. 2011;60(21):705-712.

24. Centers for Disease Control and Prevention. Update: influenza activity - United States, 2011-12 season and composition of the 2012-13 influenza vaccine. MMWR Morb Mortal Wkly Rep. 2012;61(22):414-420.

25. Influenza activity-United States, 2012-13 season and composition of the 2013-14 influenza vaccine. MMWR Morb Mortal Wkly Rep. 2013;62(23):473-479.

26. Epperson S, Blanton L, Kniss K, et al. Influenza activity - United States, 2013-14 season and composition of the 2014-15 influenza vaccines. MMWR Morb Mortal Wkly Rep. 2014;63(22):483-490.

27. Appiah GD, Blanton L, D'Mello T, et al. Influenza activity United States, 2014-15 season and composition of the 201516 influenza vaccine. MMWR Morb Mortal Wkly Rep. 2015;64(21):583-590.

28. Lindsay L, Jackson LA, Savitz DA, et al. Community influenza activity and risk of acute influenza-like illness episodes among healthy unvaccinated pregnant and postpartum women. Am J Epidemiol. 2006;163(9):838-848.

29. McGrath LJ, Kshirsagar AV, Cole SR, et al. Influenza vaccine effectiveness in patients on hemodialysis: an analysis of a natural experiment. Arch Intern Med. 2012;172(7):548-554.

30. Cuthbertson CC, Kucharska-Newton A, Faurot KR, et al. Controlling for frailty in pharmacoepidemiologic studies of older adults: validation of an existing Medicare claims-based algorithm. Epidemiology. 2018;29(4):556-561.

31. Faurot KR, Jonsson Funk M, Pate V, et al. Using claims data to predict dependency in activities of daily living as a proxy for frailty. Pharmacoepidemiol Drug Saf. 2015;24(1):59-66.

32. Brookhart MA, Patrick AR, Dormuth $C$, et al. Adherence to lipidlowering therapy and the use of preventive health services: an investigation of the healthy user effect. Am J Epidemiol. 2007;166(3):348-354.

33. Shrank WH, Patrick AR, Brookhart MA. Healthy user and related biases in observational studies of preventive interventions: a primer for physicians. J Gen Intern Med. 2011;26(5):546-550.

34. Lau B, Cole SR, Gange SJ. Competing risk regression models for epidemiologic data. Am J Epidemiol. 2009;170(2):244256.

35. Fine J, Gray R. A proportional hazards model for the subdistribution of a competing risk. J Am Stat Assoc. 1999;94(446):496-509.

36. Cole SR, Hernan MA. Adjusted survival curves with inverse probability weights. Comput Methods Programs Biomed. 2004;75(1):45-49.
37. Brookhart MA, Wyss R, Layton JB, Sturmer T. Propensity score methods for confounding control in nonexperimental research. Circ Cardiovasc Qual Outcomes. 2013;6(5):604-611.

38. Yang D, Dalton JE. A unified approach to measuring the effect size between two groups using SAS. Paper presented at: SAS Global Forum; 2012; Orlando, FL.

39. Ding P, VanderWeele TJ. Sensitivity analysis without assumptions. Epidemiology. 2016;27(3):368-377.

40. R Core Team. R: A language and environment for statistical computing, Vienna, Austria. 2014. http://www.R-project.org/. Accessed December 18, 2018.

41. Austin PC. Balance diagnostics for comparing the distribution of baseline covariates between treatment groups in propensityscore matched samples. Stat Med. 2009;28(25):3083-3107.

42. Centers for Medicare \& Medicaid Services. Seasonal influenza vaccines pricing. https://www.cms.gov/Medicare/MedicareFee-for-Service-Part-B-Drugs/McrPartBDrugAvgSalesPrice/ VaccinesPricing.html. Accessed December 12, 2018.

43. Saran R, Robinson B, Abbott KC, et al. US Renal Data System 2018 Annual Data Report: epidemiology of kidney disease in the United States. Am J Kidney Dis. 2019;73(3)(suppl 1): A7-A8.

44. Falsey AR, Treanor JJ, Tornieporth N, Capellan J, Gorse GJ. Randomized, double-blind controlled phase 3 trial comparing the immunogenicity of high-dose and standard-dose influenza vaccine in adults 65 years of age and older. J Infect Dis. 2009;200(2):172-180.

45. DiazGranados CA, Dunning AJ, Robertson CA, Talbot HK, Landolfi V, Greenberg DP. Effect of previous-year vaccination on the efficacy, immunogenicity, and safety of high-dose inactivated influenza vaccine in older adults. Clin Infect Dis. 2016;62(9):1092-1099.

46. DiazGranados CA, Saway W, Gouaux J, et al. Safety and immunogenicity of high-dose trivalent inactivated influenza vaccine in adults 50-64 years of age. Vaccine. 2015;33(51): 7188-7193.

47. Kaka AS, Filice GA, Myllenbeck S, Nichol KL. Comparison of side effects of the 2015-2016 high-dose, inactivated, trivalent influenza vaccine and standard dose, inactivated, trivalent influenza vaccine in adults $>/=65$ years. Open Forum Infect Dis. 2017;4(1):Ofx001.

48. Moro PL, Arana J, Cano M, et al. Postlicensure safety surveillance for high-dose trivalent inactivated influenza vaccine in the Vaccine Adverse Event Reporting System, 1 July 2010-31 December 2010. Clin Infect Dis. 2012;54(11): 1608-1614.

49. McGrath LJ, Ellis AR, Brookhart MA. Controlling timedependent confounding by health status and frailty: restriction versus statistical adjustment. Am J Epidemiol. 2015;182(1):17-25.

50. Vogtlander NP, Brown A, Valentijn RM, Rimmelzwaan GF, Osterhaus AD. Impaired response rates, but satisfying protection rates to influenza vaccination in dialysis patients. Vaccine. 2004;22(17-18):2199-2201.

51. Scharpe J, Peetermans WE, Vanwalleghem J, et al. Immunogenicity of a standard trivalent influenza vaccine in patients on long-term hemodialysis: an open-label trial. Am J Kidney Dis. 2009;54(1):77-85.

52. Kunisaki KM, Janoff EN. Influenza in immunosuppressed populations: a review of infection frequency, morbidity, mortality, and vaccine responses. Lancet Infect Dis. 2009;9(8): 493-504.

53. Brydak LB, Roszkowska-Blaim M, Machala M, Leszczynska B, Sieniawska M. Antibody response to influenza immunization in 
two consecutive epidemic seasons in patients with renal diseases. Vaccine. 2000;18(28):3280-3286.

54. Evison J, Farese S, Seitz M, Uehlinger DE, Furrer $H$, Muhlemann K. Randomized, double-blind comparative trial of subunit and virosomal influenza vaccines for immunocompromised patients. Clin Infect Dis. 2009;48(10):1402-1412.

55. Broeders NE, Hombrouck A, Lemy A, et al. Influenza A/ $\mathrm{H} 1 \mathrm{~N} 1$ vaccine in patients treated by kidney transplant or dialysis: a cohort study. Clin J Am Soc Nephrol. 2011;6(11): 2573-2578.

56. Dikow R, Eckerle I, Ksoll-Rudek D, et al. Immunogenicity and efficacy in hemodialysis patients of an ASO3(A)-adjuvanted vaccine for 2009 pandemic influenza $A(\mathrm{H} 1 \mathrm{~N} 1)$ : a nonrandomized trial. Am J Kidney Dis. 2011;57(5):716-723.

57. Jackson LA. Benefits of examining influenza vaccine associations outside of influenza season. Am J Respir Crit Care Med. 2008;178(5):439-440.

58. Jackson LA, Jackson ML, Nelson JC, Neuzil KM, Weiss NS. Evidence of bias in estimates of influenza vaccine effectiveness in seniors. Int J Epidemiol. 2006;35(2):337-344.

59. Jackson LA, Nelson JC, Benson P, et al. Functional status is a confounder of the association of influenza vaccine and risk of all cause mortality in seniors. Int J Epidemiol. 2006;35(2):345352.

60. Jackson ML, Yu O, Nelson JC, et al. Further evidence for bias in observational studies of influenza vaccine effectiveness: the 2009 influenza $\mathrm{A}(\mathrm{H} 1 \mathrm{~N} 1)$ pandemic. Am J Epidemiol. 2013;178(8):1327-1336.

61. Butler AM, Layton JB, Krueger WS, Kshirsagar AV, McGrath LJ. Assessing residual bias in estimating influenza vaccine effectiveness: comparison of high-dose versus standard dose vaccines. Med Care. 2019;57(1):73-78.

62. Centers for Disease Control and Prevention. Vaccine effectiveness - how well does the flu vaccine work?. https://www. cdc.gov/flu/about/qa/vaccineeffect.htm. Accessed December $12,2018$.

63. Biggerstaff M, Kniss $K$, Jernigan DB, et al. Systematic assessment of multiple routine and near real-time indicators to classify the severity of influenza seasons and pandemics in the United States, 2003-2004 through 2015-2016. Am J Epidemiol. 2018;187(5):1040-1050.

64. Allen JD, Ross TM. H3N2 influenza viruses in humans: viral mechanisms, evolution, and evaluation. Hum Vaccin Immunother. 2018;14(8):1840-1847. 